preparation of sterile solutions of procaine hydrochloride. The author's conclusions are that sterile solutions can be prepared without the use of bacteriaproof filters, provided aseptic methods are used. Solutions prepared with an antiseptic present are not vulnerable to contamination likely to occur in a laboratory devoted to the production of sterile preparations. Solutions prepared with both an antiseptic present and an oxidation-preventive substance such as sulphur dioxide are even less vulnerable to contamination. The antiseptic properties of solutions of procaine hydrochloride tend to increase with increase of percentage strength.

A second paper, by G. E. Shaw and H. G. Hind, from the Evans Biological Institute, was on "Some Aspects of the Storage of Antitoxins During Wartime". Antitoxic serum, the blood serum of hyperimmunized animals, is issued for sale either in its natural state or, more usually, as a solution of a selected protein fraction therefrom; it is of special importance in war-time owing to the exposure of the population to risk from epidemic disease and the occurrence of a large number of injuries which necessitate antitetanic treatment. Antitoxin is stored in a sterile condition at about $4^{\circ} \mathrm{C}$. in large glass containers. In view of the risk from damage through enemy aerial activity, bulk storage presents a serious problem, owing to the possibility of containers breaking and the supply of electricity to the refrigerator plant being interrupted. The authors sought for a method which would eliminate the need for glass containers, and make the use of refrigeration unnecessary, but would, at the same time, preserve the potency and maintain the sterility of the product. They described two methods, each yielding a dry powder which may be stored in any type of container. The potency of the product is maintained. Cold-storage accommodation is unnecessary, and the powder is self-sterile. The desiccation method requires special apparatus for drying, but this does not involve a large capital outlay. The material contains so much preservative that, although reasonable care in handling is naturally adopted, complete asepsis is not essential.

\title{
PHOTOPHORES IN DECAPOD CRUSTACEA
}

$\mathrm{D}^{\mathrm{R}}$ R. DENNELL'S work on the structure of the photophores in certain decapod crustacea from deep water is of outstanding interest and importance*. It has been known for long that luminous organs exist in many of the deep-sea decapods; but it is astonishing to find such a variety of structure and so much difference in closely related forms.

The photophores described in this work are not all proved luminous organs, but morphological evidence makes it extremely likely that this is so. Only in a few cases have live animals been seen to emit light, but those who have observed the phenomenon have made careful notes of the position of the light organs, and in these cases it is certain that true photophores are present. The reason for the luminescence is still obscure. As in the squids and fish which possess them, the luminous organs mostly throw their light downwards, lighting an area outside the field of vision, it is especially difficult to understand why in nearly all species of Sergestids examined, and also in Parapandalus, some of the photophores should be situated in such a position that they must illumine the branchial chamber and throw light downwards on the gills. There is nothing in the structure of the gills themselves to show that they could benefit from such illumination, and it may be as is suggested (but with serious objections) that the development of light-organs here is simply the response to a rich oxygen supply.

Within the genus Sergestes no fewer than four different types of photophores are found : (1) The organs of Pesta which occur in the larger number of species and are modified liver tubules of endodermal origin; (2) complex organs with lenses scattered superficially on the body which are found in Sergestes challengeri and its allies; (3) superficial simple organs without lenses scattered over the body as in $S$. regalis (this being an entirely new type of luminous organ); (4) simple superficial streaks on the carapace which are found to be present in those species which also possess the organs of Pesta. The second and

* On the Structure of the Photophores of some Decapod Crustacea. By Dr. Ralph Dennell. Discovery Reports, 20, 307-382, plates XXIV-XXVI (1940). fourth of these are of ectodermal origin, the third may be ectodermal, or, possibly, mesodermal. It is shown that the structure of the photophores in the various species of Sergestes may be entirely different and that it is unsafe to conclude that any are similar unless the minute structure is examined, even if they are in the same position.

In Parapandalus richardi, which with some other pandalids possesses photophores outwardly like the main bodies of the organs of Pesta of Sergestids, these organs have a totally different minute structure. It is a remarkable fact that such similar organs should have been separately evolved in the two groups; it shows that they must subserve some important function, if, as yet, not sufficiently understood. There are also in Parapandalus organs on the pleopods which are doubtfully designated as photophores.

In the Hoplophorids the photophores have reached a very high state of complexity, and here again a variety of types is shown, movement being well developed. The pleopod organs are very similar in all the species of Hoplophorus and Systellaspis examined, but the other types occur very irregularly ; photophores in similar positions in two species are frequently entirely different in structure, while in a single species a number of different types of organ is to be found. For the most part the various kinds of organ are entirely distinct and they do not form stages in a developmental series. It is a remarkable fact that the luminous streak behind the fifth leg, which is a conspicuous feature in both Systellaspis and Hoplophorus, should have an entirely different structure in these two closely related genera.

The present investigation is a purely morphological one, but it is impossible to describe these organs of such unusual diversity without touching on their probable function and methods of working. The remarks made are suggestive and cry aloud for more work on this extremely interesting subject.

In conclusion, tribute must be paid to the very excellent technique and quality of the illustrations throughout. 